\title{
A Black Swan Event Drives Eco-Evolutionary Heterogeneity
}

\author{
Denon Start $^{1}$ \\ ${ }^{1}$ University of Toronto
}

May 5, 2020

\begin{abstract}
Environmental variation is a constant. Difficult to predict but important 'Black Swan' events are increasing in frequency and magnitude, but we are only beginning to understand the ecological and evolutionary consequences of such events. Extreme events can increase or decrease eco-evolutionary heterogeneity depending on the spatial grain at which they occur. Here I present a 6-year study of $3000+$ individual univoltine gall makers and their enemies from 15 populations. An extreme event in one generation homogenized a key environmental determinant of enemy attack rates and survival, but exposed gall makers to an alternative environmental driver of ecological interactions. Counterintuitively, rather than acting as an ecological or evolutionary filter, extreme events can create greater spatial variation in demography, species interactions, natural selection, and evolutionary change. I suggest that the eco-evolutionary consequences of Black Swan events can only be understood by considering the evolutionary outcome of what are often complex species interactions.
\end{abstract}

\section{Introduction}

'For whoever knows the ways of Nature will more easily notice her deviations; and, on the other hand, whoever knows her deviations will more accurately describe her ways'

-Francis Bacon in Novum Organum, 1620

Neither the biotic nor abiotic environment are fixed - they are an ever-changing milieu of shifting conditions, traits, and abundances. While environmental variation may be regular and predictable (e.g. seasonality), there are also rare, unpredictable, and large environmental pulses (e.g. hurricanes; Ackerly 2003, Nunez and Logares 2012, Anderson et al. 2017). These extreme "Black Swan" events are increasingly recognized as biologically important, but we know relatively little about how these extreme events shape ecological and evolutionary processes, especially in the wild (Pruitt et al. 2019). Understanding how extreme Black Swan events shape ecological and evolutionary systems is a particularly pressing issue as such events appear to be increasing in frequency (Fey et al. 2015), a pattern predicted by models of global change.

Extreme events impose harsh ecological and evolutionary filters, potentially triggering large demographic changes, shifts in community composition, and strong natural selection (Fey et al. 2015, Siepielski and Benkman 2007, Little et al. 2019). For instance, rare but regular fire events can reset succession by removing most trees from a forest (Shafi and Yarranton 1973), while also strongly favoring trees with fire-resistant traits (Perry and Lotan 1979). Extreme events can also create characteristic patterns across broader spatial scales relevant to the maintenance of biodiversity. When extreme events occur in some but not other areas, they create a mosaic of abiotic heterogeneity (Siepielski and Benkman 2007, Little et al. 2019). However, when extreme events occur simultaneously over large areas (e.g. a hurricane hitting a whole island), it is generally assumed that the environment, and the corresponding ecological interactions and patterns of selection, will be homogenized (Little et al. 2019). These hetero/homo-genization of abiotic conditions caused by Black Swan events can have key landscape-level consequences for ecological and evolutionary patterns. If the environment is homogenized, it should reduce $\beta$-diversity, spatial variation in natural selection, and the evolutionary trajectory of populations. Conversely, extreme events that create abiotic and biotic heterogeneity may be 
crucial for maintaining biological diversity across a landscape by allowing species with different requirement to use different patches, and by creating divergent patterns of natural selection and evolutionary change (Start et al. 2020). Extreme events should influence landscape level patterns of environmental conditions and corresponding eco-evolutionary change.

Most ecological and evolutionary research is focused on investigating regular and predictable events, rather than extreme ones. The dearth of research exploring the consequences of extreme events is surprising given their likely importance (Ackerly 2003, Fey et al. 2015, Pruitt et al. 2019), but may stem from some key methodological constraints (Nunez and Logares 2012). One key issue is that, while some events like hurricanes can be described as rare and extreme events post-hoc (Pruitt et al. 2019), in most cases understanding what constitutes an extreme event requires the long-term monitoring of a biological system - a 100 year storm for one population may be a common occurrence for another. While long term datasets do exist, they generally focus on one or few communities, do not distinguish among what may be distinct population (Nunez and Logares 2012), and/or do not measure intraspecific variation (but see Pruitt et al. 2019 and Siepielski and Benkman 2013), crucial data for understanding eco-evolutionary change. To understand the broader spatial effects of Black Swan events therefore requires both long-term monitoring of species' abundances and individual traits, and the replication of surveys across multiple distinct communities and populationsfew such datasets exist. Because the landscape-level effects of extreme events likely shape eco-evolutionary dynamics, we require long-term spatially replicated datasets to gain a better understanding of the forces structuring biological systems.

Here, I present a 6-year dataset spanning 15 populations that describes the interactions between a gallmaking fly and its enemies. Gall size is under selection-bird attack favors small galls, parasitoid attack favors large galls, and the net direction of selection depends on the balance of both attack rates (Abrahamson 1989, Start and Gilbert 2016). Bird attack rate (and resultant selection) is influenced by several key factors, notably whether a gall has been knocked to the ground (making them less salient; Start 2018a) and the distance of the gall from forest habitat where bird predators reside (Start et al. 2018). In the 5th year of monitoring, a windstorm homogenized one of these key environmental variables - virtually all galls were knocked to the ground. I ask how the homogenization of this key environmental variable affects the local and landscape consequences of (1) gall-maker survival, (2) species interactions, (3) selection, (4) demographic consequences in the following generation, and (5) evolutionary change.

\section{Methods}

\section{Study System}

Goldenrod (Solidago altissima) is a clonal patchily distributed old-field plant that is common in eastern North America. A common goldenrod herbivore is Eurosta solidaginis - a univoltine fly whose larvae spur the development of a spherical stem gall (Uhler 1951). Eurosta emerges as an adult in late May in Southern Ontario, mates, and oviposits gall-inducing larvae into the apical meristem of fresh goldenrod shoots in the following $\sim 2$ weeks. Gall size is a complex function of fly genotype, plant genotype, and environmental conditions, but adaptive shifts in gall size corresponding to patterns of selection have been observed (Craig et al. 2007, Start and Gilbert 2016).

Selection and resultant adaptive evolution stems from attack by enemies of Eurosta, including Mordellistena beetle larvae, two parasitoid wasps (Eurytoma spp. ), and birds (downy woodpeckers and chickadees). Eurytoma gigantea and birds also attack galls of different sizes, preferring to forage on small and large galls respectively (Abrahamson et al. 1989). As a result, selection on gall size tends to favor intermediate forms (stabilizing selection) but can also have a directional component favoring the size favored by the rarer enemy. These victim-enemy interactions vary considerably through time (Weis and Kapelinski 1994), and across space (Craig et al. 2007, Start et al. 2018, Start 2019), including at very small spatial scales (Start and Gilbert 2016, Start et al. 2019).

Beyond preferences for different sized galls, the enemies ofEurosta also differ in phenology. Beetle larvae and parasitoid wasps attack galls in early summer while the gall is still growing and goldenrod tissue is still 
alive, whereas bird attack predominantly occurs over winter after the plant tissue has died but while the larvae remains alive inside the gall. During the late fall, it is common for some dead goldenrod stems (but that nevertheless have livingEurosta in them) to be knocked over in windstorms. Because birds prefer to attack galls higher off the ground (Start 2018a), galls on knocked-over stems will likely experience lower bird predation, causing selection to be more positive (favor larger galls). In the rare event that windstorms knock down all stems and galls on the landscape, then we would expect bird predation to be homogenous and low, and selection to be consistent among populations and positive.

\section{Survey}

I began a long-term survey of 15 populations of goldenrod in Spring 2015. I demarcated populations at the Koffler Scientific Reserve (KSR), Ontario, Canada with the criteria that (i) goldenrod (and thusEurosta) were in distinct patches separated from one another by a matrix of grass and other forbs, (ii) populations were separated by at least $50 \mathrm{~m}$ to ensure relative population isolation (Cronin et al. 2001, Start and Gilbert 2016), and (iii) populations were broadly distributed across the entire study site (see map of locations in Fig. S1).

Beginning in 2015 I collected up to 50 galls per population annually (Table S1). All galls were collected in mid-May within $5 \mathrm{~m}$ of the centre marker of each population. For each gall collected I began by measuring horizontal diameter. I then scored each gall containing Eurostaas a survivor, those with large holes as having been killed by a bird, and those containing other larvae as having been killed by the corresponding enemy (e.g. Eurytoma gigantea ). I scored empty galls with no sign of habitation as early larval death (Abrahamson et al. 1989). Note that mortality is often high (Weis and Kapelinski 1994, Start 2018b), suggesting that survival is a key fitness component. Further note that gall size is unrelated to larval mass or size of the adult fly (Hess et al. 1996), suggesting that gall size is unlikely to be correlated with other components of fitness like fecundity or mating success.

In addition to measuring individual gall size and species interactions, I also collected key environmental information. I recorded the number of stems out of 100 randomly selected goldenrod that were knocked down and the distance of the population from trees. All three metrics have previously been shown to influence bird attack, and in some cases selection. Furthermore, I estimated density by counting the number of galls on 100 randomly selected goldenrod stems - density should increase following years with high survival but decline following years when enemy attack was high.

In late June 2019, I measured the density (galls per goldenrod stem) of galls in each patch, and measured the horizontal diameter of up to 20 galls per population. Note that these measurements were taken on the 2019/2020 generation, and were taken to measure the ecological (demographic) and evolutionary (trait change) consequences of the previous year's extreme event (i.e. that occurred in the 2018/19 generation). Together these data represent demographic information (survival and density), species interactions (attack rates), and the fodder to measure natural selection and evolutionary change (trait values).

Statistical Analyses

In fall 2018 (the 2018/19 generation) a large wind event seemed to knock down most goldenrod stems, and correspondingly most galls. The goal of my analyses were to (i) confirm that event as having an extreme effect on environmental conditions, (ii) explore corresponding patterns of mortality, demography, and enemy attack, including mean changes and differences among populations, and (iii) test for corresponding differences in natural selection and evolutionary change.

\section{Environmental Correlates}

I began by investigating changes in the percentage of stems and galls knocked over among years. Note that while the extreme weather event occurred in 2018, the affected gall collection occurred in 2019 and is referred to as such (i.e. the 2018 extreme event affected the 2018/19 generation). I began by using a linear mixed model (LMM) to test for differences in the ln-transformed percentage of stems knocked down across years, including year as a factor and population as a random effect. This model is designed to test for 
changes in the mean of a key environmental condition. However, extreme events can also change patterns of environmental variation among populations and communities. As such, I next used a Levene's test to test for difference in variance among pairwise combinations of years. As above, I controlled for consistent population-level differences using a random effect. Together these two tests quantify the mean change and among-population variance change in stem knock down.

\section{Ecological Interactions}

After quantifying the environmental effects of the storm, I next sought to characterize the consequences of those environmental differences for survival and species interactions. Because attack byMordellistena and Eurytoma occurs in summer, they should be unaffected by galls having been knocked to the ground. Conversely, bird attack is likely to decline if galls are knocked to the ground and are therefore less high off the ground (Start 2018a), and also tends to decline further away from forest edges where woodpeckers live (Start et al. 2018). I tested for these effects in two ways. First, I used an LMM to estimate total mortality and attack rates of each enemy independently, including year as a factor and population as a random effect. I then performed pairwise comparisons of attack rates among years using Tukey's tests. Second, I used another LMM to estimate the same measures of mortality and attack rates, but while including distance to trees and percentage of stems knocked down as main effects. In combination, these two tests (i) estimate among year differences in demography and species interactions, and (ii) tie any differences to specific environmental conditions.

While the above tests quantify mean differences among years and environmental conditions, they do not estimate among-population variation (i.e. whether the extreme event homogenizes the environment or creates environmental variation). I again tackled this issue using Levene's tests. I independently tested for differences in among-population but within-year variation in mortality and each attack rate. I again compared years using Tukey's tests to gain pairwise comparisons. I also tied differences in variable species interactions to environmental variation. Specifically, I calculated residuals for each environmental variable and each ecological interaction. Residuals were measured from the grand mean across all populations, but within a given year. I then used linear models to estimate the residuals of each species interaction using residual variance in the distance from trees and the residual variance in the percentage of stems knocked down. As above, this combination of tests quantifies among-population variance in species interactions while tying that variation to corresponding environmental variation.

\section{Natural Selection}

The combination of bird and Eurytoma gigantea attack have well known consequences for patterns of selection. When bird attack is more common, selection is negative and favors small galls. WhenEurytoma gigantea attack is common, selection is positive and larger galls are more likely to survive. Accordingly, environmental changes resulting in differences in average attack rate of one or both enemies may change average patterns of selection, and changes to the among-population variation in attack rates should alter among-population differences in the direction and magnitude of selection.

Before relating selection to extreme events or other environmental drivers, I first had to estimate selection in each population. I began by standardizing gall size by subtracting the population mean gall size and dividing by the standard deviation. I similarly relativized fitness by dividing survival (zero or one) by mean survival in the population in that year. Standardizing and relativizing locally assumes soft selection and allows us to compare estimates of selection among populations that differ in mean fitness. After standardizing variables, I calculated a selection coefficient for each year by population combination by regressing individual relative fitness against standardized gall size in a linear model (Lande and Arnold 1983). Note that while data are binary, generalized linear models do not allow for the estimation of standard parameter values commonly used in selection analyses (e.g. selection gradients), requiring the use of linear models.

After calculating selection gradients, I began testing for the effects of extreme events and environmental conditions on natural selection. First, I used a series of linear models to estimate selection coefficients. I began by running an analysis testing for among-year differences while including population as a random effect, 
applying Tukey's tests to make pairwise comparisons. I continued by running a separate model including bird and Eurytoma attack rates as main effects. These analyses then test for relationships between selection and increasingly distant processes (e.g. windstorm in a particular yearenvironmental conditionsecological interactionsselection).

I next sought to quantify spatial variation in selection. I first used Levene's tests to estimate within-year but among-population difference in selection coefficients, again using Tukey's tests to make pairwise comparisons. I continued this analysis by comparing within-year residual variation in selection coefficients to residual variation in bird attack and survival as main effects. Recall that bird attack shapes the fitness surface, while survival rates are our measure of mean fitness and can therefore inflate or dampen the opportunity for selection and thus the magnitude of selection coefficients (Arnold and Wade 1984, Benkman 2013).

\section{Ecological and Evolutionary Consequences}

I tested for two potential consequences of the 2018/19 extreme wind event-one ecological and one evolutionary. I began by investigating the demographic (ecological) consequences of the 2018/19 extreme wind event. First, I used an LMM with year as a main effect and patch as a random effect to estimate gall density in June 2019 (i.e. the generation after the extreme event). I then used a Levene's test to compare spatial variation in gall density among years.

I next moved on to the potential evolutionary consequences of the 2018/19 wind event. Because galls tend to change size from June to the following May (but reaction norms are parallel; Weis and Gorman 1990), I began by calculating the difference in gall size between the 2018/19 generation measured in May 2019 and the 2019/20 generation measured in June 2019 (i.e. the 5th and 6th generations). I then standardized this trait change, and used standardized trait change as the response variable in a LM with selection coefficients as the main effect.

Note that in all above analyses I carried error associated with selection estimates forward into subsequent analyses by weighting those analyses by the inverse of the standard error associated with each estimate. All analyses were conducted in R using the 'Ime4' (Bates et al. 2015) and 'car' (Fox and Weisberg 2011) packages.

\section{Results}

An extreme wind event in 2018/19 caused the homogenization of a key environmental variable while exposing Eurosta populations to other environmental differences, ultimately creating biotic heterogeneity and correspondingly variable selection. The wind event of 2018/19 knocked down 96\% of all goldenrod stems (compared to $51 \%$ in the next most extreme year; $\mathrm{P}<0.001$ pairwise differences between 2019 and any other years), reducing spatial variation in this key environmental factor (Fig. 1A; $\mathrm{P}<0.002$ for all pairwise differences between 2019 and other years).

Consistent with a higher proportion of stems being knocked over, bird attack was less frequent for the 2018/19 generation (21\% attack rate; $\mathrm{P}<0.001$ for all 2019-other year contrasts, except $\mathrm{P}=0.16$ for 2019 versus 2017). However, spatial variation in bird attack rate was actually greater in 2019 than other years (Fig. 2A; $\mathrm{P}<0.03$ in all pairwise comparisons). This counterintuitive result occurred because when most stems were knocked down, the distance of a population from trees (another source of environmental variation) became an important determinant of bird attack (Fig. 2B). When most stems remain standing, bird attack rate does not vary among populations. However, when most stems are knocked down, bird attack is greater near the trees (Stems knocked down $\mathrm{x}$ distance to trees interaction: $\mathrm{P}=0.001$ ).

No other enemy differed in mean or spatial variance among years (all $\mathrm{P}>0.2$ ), and as expected attack rates of other enemies were unaffected by stems being knocked over or by the distance of the population from the tree line (all $\mathrm{P}>0.25$ ). Patterns of mortality were consistent with the effects of year on the attack rates of particular enemies. Mortality was lowest in 2019 (Fig. 1A; 55\% compared to an average of $72 \%$ in other years; $\mathrm{P}<0.003$ in all pairwise combinations except 2019 versus 2017 where $\mathrm{P}=0.10)$. Conversely, spatial 
variation in mortality was most variable in 2019 (Fig. 1A; $\mathrm{P}<0.03$ in all pairwise combinations except 2019-2018 where $\mathrm{P}=0.19$ ).

These patterns of survival and biotic interactions shaped differences in selection. In terms of mean directional selection, 2019 was not an exceptional year. The mean $\beta$-value of +0.09 fell within the range of directional selection observed in other years (e.g. $\beta=0.16$ in 2017 and $\beta=-0.22$ in 2015), and only differed significant from 2015 (Fig. 3A; $\mathrm{P}=0.001$, all other pairwise comparisons $\mathrm{P}>0.5$ ). However, 2019 did exhibit exceptionally variable patterns of selection. Spatial variation in directional selection was greater in 2019 than in all other years (Fig. 3B; variance in $2019=0.31$, average variance in other years $=0.19$, all $\mathrm{P}<0.03$ ). The increased spatial variation in selection was partly driven by more variable bird attack (changing the shape of the fitness surface) and partly by more variable survival (Fig 3B; stretching and compressing the fitness surface; interaction term: $\mathrm{P}=0.002$ ). By exposing gall-makers to alternative environmental variation, the windstorm of 2018/19 created biotic heterogeneity in attack and survival, and corresponding spatial variation in patterns of natural selection.

Heterogeneity in survival and selection created corresponding ecological and evolutionary consequences in the subsequent generation. Specifically, densities in the $2019 / 20$ generation increased as a result of reduced mortality in the 2018/19 generation (all pairwise comparisons $\mathrm{P}<0.04$ ), but densities tended to be more spatially variable $(\mathrm{P}<0.03$ for all pairwise comparisons except $2019-2017$ where $\mathrm{P}=0.15)$. Finally, gall sizes exhibited a likely evolutionary response to selection - galls tended to increase or decrease in size for positive versus negative directional selection coefficients respectively (Fig. 4; $\mathrm{P}=0.04$ ). In short, an extreme event created biotic heterogeneity with ecological and evolutionary consequences.

\section{Discussion}

Extreme events are increasingly common and can have corresponding consequences for ecological (Fey et al. 2017, Anderson et al. 2017) and evolutionary (Siepielski and Benkman 2007, Little et al. 2019) processes. However, the non-local eco-evolutionary consequences of extreme events have not been explored. I show that an extreme event has different consequences for species interactions, selection, demography, and evolution in different populations (all Figures). Consequently, the extreme event increased heterogeneity of species interaction ( $\beta$-diversity; Fig. 2) and spatial variation in selection (Fig. 3), with down-stream eco-evolutionary consequences (Fig. 5). We know that extreme events can have important consequences for local ecological and evolutionary dynamics. The work presented here shows that Black Swan events can also create characteristic landscape-level patterns of eco-evolutionary heterogeneity, with potential importance for the maintenance of regional biodiversity.

The extreme wind event had key consequences for survival and species interaction. The extreme event described here is somewhat unusual - the vast majority of extreme events increase death (Anderson et al. 2017), while the extreme event here reduced antagonistic interactions and thus mortality (Fig. 1A \& 2). More intriguingly, while the extreme wind event homogenized the proportion of galls being knocked over (Fig. 1A), it nevertheless increased spatial heterogeneity in survival and bird attack (Fig. 1A \& 2A). This occurred because when most stems were knocked down, the distance of a population from the treeline had a larger effect on attack rates - a mean change in one environmental condition altered the effect of another environmental condition on ecological interactions (Fig. 2B). Similar counterintuitive effects of extreme events are likely to occur whenever multiple factors interact to shape ecological processes - the complexity of interaction networks and their variations may serve to amplify, dampen, or qualitatively alter the effects of extreme events (Siepielski and Benkman 2007, Ledger et al. 2013, Woodward et al. 2016, terHorst et al. 2018). Indeed, species interactions are often influenced by multiple interacting environmental factors and can serve as 'biotic multipliers' of an environmental change (Zarnetske et al. 2012). In short, to understand the consequences of Black Swan events, it is not enough to test for the direct effects of the environmental change on an ecological or evolutionary outcome (e.g. does a heatwave increase or decrease mortality). Instead, we require a greater understanding of how extreme events shape species interactions (Siepielski and Benkman 2007), and the consequences of those interaction changes for populations and communities. 
Extreme events can have direct and indirect consequences on patterns of natural selection. In the simplest case, an extreme event can directly select for different phenotypes. For instance, selection induced by tropical cyclones favors aggressiveness in colonies of spiders (Little et al. 2019). Conversely, extreme events can alter selection by having cascading effects on a focal population via species interactions (Siepielski and Benkman 2007). In our case, the greater heterogeneity in bird attack and survival created by the extreme wind event caused correspondingly variable patterns of natural selection (Fig. 3). Interestingly, this pattern is opposite to what is traditionally expected - extreme events occurring across all populations should homogenize interactions and selection, while extreme events occurring in some but not other local communities should create heterogeneity (Pruitt et al. 2019). As a result, extreme events may impose strong and highly variable selection. While strong selection in some years can drive patterns of local adaptation (Siepielski and Benkman 2007), greater spatial variation in selection can exacerbate among-population differences and potentially contribute to the regional maintenance of genetic and phenotypic diversity. As above, we cannot understand the causes of selection in response to extreme events without considering species interactions and indirect environmental effects in multiple populations.

The effect of Black Swan events on ecological and evolutionary change are linked and complex. Extreme events should have a large impact on survival and population density in the next generation, creating a correspondingly strong pattern of selection and evolutionary change (Benkman 2013). Indeed, following the high survival associated with the extreme wind event, population density of Eurosta increased. Large differences in mean survival also drove evolutionary change galls tended to increase or decrease in size in line with the direction of selection (Fig. 4) which itself was created by differences in survival and bird attack (Fig. 3B). On the one hand, it is unsurprising that multiple ecological quantities (survival and species interactions) affected selection and evolutionary change (Arnold and Wade 1984, Benkman 2013). On the other hand, it does suggest a need to simultaneously account for population and community dynamics when forecasting evolutionary change (terHorst et al. 2018, Start et al. 2019), including in response to extreme events.

Here I have demonstrated an eco-evolutionary response to an extreme event, but are such responses common and important? The answer to the first question is pedantic - extreme events are almost definitionally rare, so eco-evolutionary responses to such events should be correspondingly uncommon (Anderson et al. 2017). However, when rare extreme events do occur, I argue that they can have important consequences for ecoevolutionary processes. For instance, extreme events can trigger extinctions (Nunes and Logares 2012), alter community structure (Shafi and Yarranton 1973), shift population dynamics (Anderson et al. 2017), and drive patterns of adaptation (Siepielski and Benkman 2007). In some instances, this occurs because extreme values have an outsized effect on eco-evolutionary patterns. For instance, the long run fitness (and frequency) of a given genotype depends on geometric mean fitness, inflating the importance of rare but extreme events that affect fitness in some generations (Saether and Engen 2015). However, the effects of extreme events may also stem from other mechanisms, for instance by triggering permanent extirpations or tipping a system into an alternative stable state (Holmgren et al. 2006, Fabina et al. 2015). I argue that extreme events should have strong eco-evolutionary consequences, but that we need more long-term datasets to gain a better understanding of the mechanisms translating a Black Swan to an eco-evolutionary outcome.

I have demonstrated that extreme events can have counterintuitive effects on eco-evolutionary heterogeneity. There are two key-takeaways from my work. First, in order to understand the effects of Black Swan events on population demography and evolutionary change, we need to understand how the multiple interactive effects of environmental factors and species interactions shape selection; interaction networks can amplify, dampen, or qualitatively alter the eco-evolutionary consequences of extreme events. Second, because of the complex interactions among species and environmental factors shaping selection and evolutionary responses, extreme events can create spatially heterogeneous eco-evolutionary patterns, even when those extreme events homogenize abiotic conditions. While it is tempting to view Black Swan events as a harsh filter with homogenizing effects (Shafi and Yarranton 1973, Nunes and Logares 2012), the complexity of nature belies such generalization. In order to understand the consequences of Black Swan events, we need to integrate our understanding of community ecology and evolutionary biology. 


\section{Acknowledgements}

I wish to thank Ben Gilbert and Art Weis for advice and financial support, and the staff and students of the Koffler Scientific Reserve for their assistance. Stephen De Lisle and Colin Bonner were key collaborators and sounding boards when beginning this work. I was variously supported by Sigma Xi, NSERC, OGS, the University of Toronto, and the Center for Population Biology (UC Davis) while collecting data and writing this manuscript.

\section{Figures}
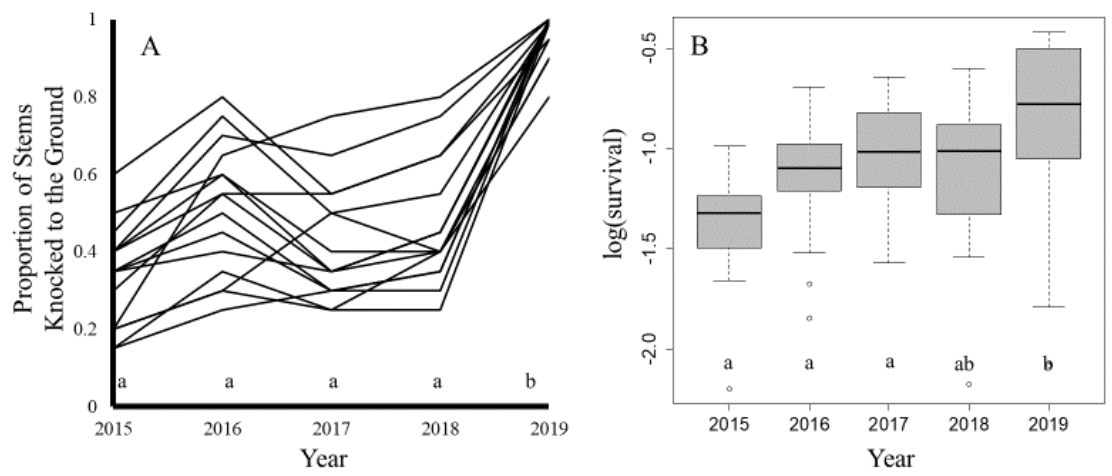

Figure 1: Patterns of environmental and demographic variability. (A) The extreme wind event of 2018/19 knocked down $96 \%$ of all stems, compared to only $50 \%$ in the next closest year. More importantly, the wind event homogenized among-patch differences in the proportion of stems and galls knocked to the ground. (B) As expected, survival increased when most galls were knocked down (and therefore free of bird predation). However, counterintuitively among-population variation in survival was greatest in the 2018/19 generation. Points in (A) each represent one year by population combination, and letters show differences in variances not means calculated using pairwise comparisons of Levene's tests.
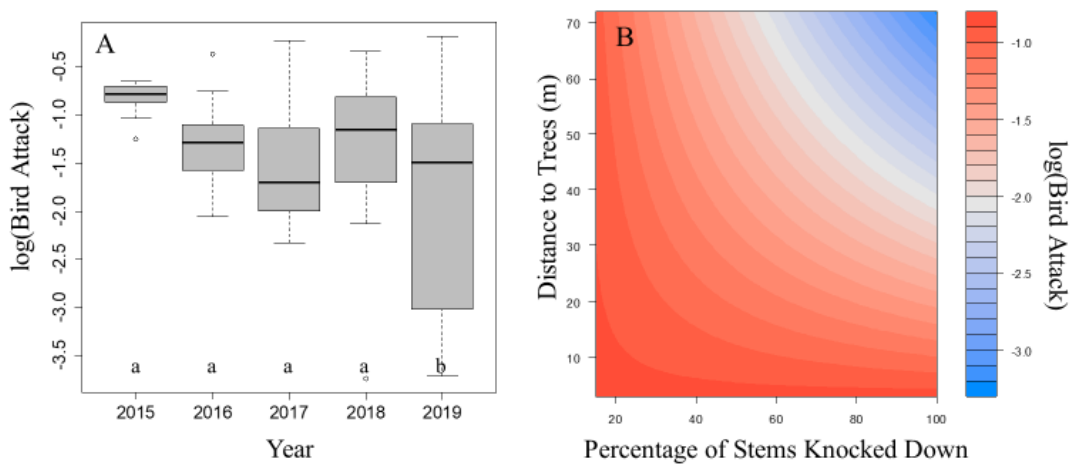

Figure 2: Patterns of species interactions and their drivers. (A) Bird attack rate was lowest in the 2018/19 
cohort. More intriguingly, spatial variation in attack rate was highest in 2018/19 despite the homogenization of a key environmental variable - the proportion of stems being knocked to the ground. (B) This counterintuitive result occurred because the homogenization of one environmental factor (stems being knocked over) exposed galls to the influence of another environmental factor (distance to trees). When galls remained on standing stems the distance of the gall from a treeline made little difference for bird attack rates, but when most stems were knocked down, birds foraged disproportionately near the treeline. Letters in (A) denote differences in variance. Colors in (B) show predicted values from a linear mixed model. Note that the percentage of stems knocked down and the distance of a population from the treeline are uncorrelated (coef $=0.002, \mathrm{P}=0.96$ in a linear mixed model).
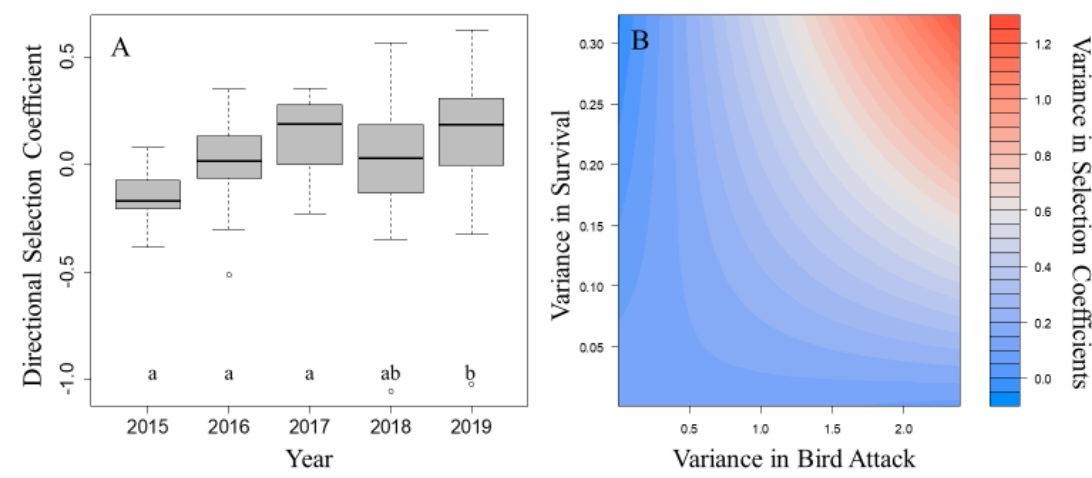

Figure 3: Patterns of selection in response to extreme weather events. (A) Mean selection varied among years, but was not remarkable in 2018/19. However, the 2018/19 cohort experienced the greatest spatial variation in selection. (B) Variation in selection was driven by a combination of spatially varying survival rates and among-population differences in bird attack rates. Letters in (A) denote variance not mean differences in selection coefficients. Color in (B) shows predicted values from a linear mixed model.

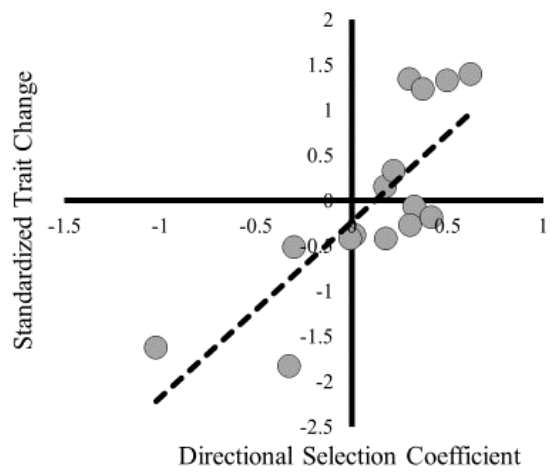

Figure 4: The evolutionary consequences of the extreme wind event. Directional selection, which was more 
variable in the 2018/19 cohort, drove trait change in the subsequent 2019/20 generation. Some populations experienced reduced gall size while other population increased their gall size.

\section{Supplementary Materials}

Table S1: The number of galls collected in each population in each year. Note that for the June 2019 measurements of the 2019/20 generation, I measured 20 individuals in all populations except the 'leek' population for which I measured 14.

\begin{tabular}{llllll}
\hline Population & Year & Year & Year & Year & Year \\
\hline & 2015 & 2016 & 2017 & 2018 & 2019 \\
bens_mantis & 51 & 43 & 57 & 50 & 53 \\
forty_east & 49 & 41 & 52 & 51 & 53 \\
forty_middle & 44 & 39 & 44 & 50 & 47 \\
forty_south_south & 50 & 40 & 52 & 50 & 52 \\
forty_west & 54 & 43 & 55 & 51 & 57 \\
greenhouse_field & 45 & 41 & 46 & 54 & 48 \\
heating_array & 48 & 40 & 49 & 50 & 51 \\
leek & 13 & 13 & 16 & 15 & 10 \\
lumberjack & 18 & 16 & 25 & 24 & 19 \\
no_name & 34 & 31 & 43 & 44 & 35 \\
opposite_office & 32 & 20 & 42 & 47 & 35 \\
shelter_north & 52 & 49 & 51 & 49 & 54 \\
shelter_pine & 50 & 44 & 49 & 50 & 52 \\
south_shelter & 29 & 37 & 40 & 43 & 33 \\
upper_potato & 67 & 43 & 60 & 53 & 62 \\
\hline
\end{tabular}

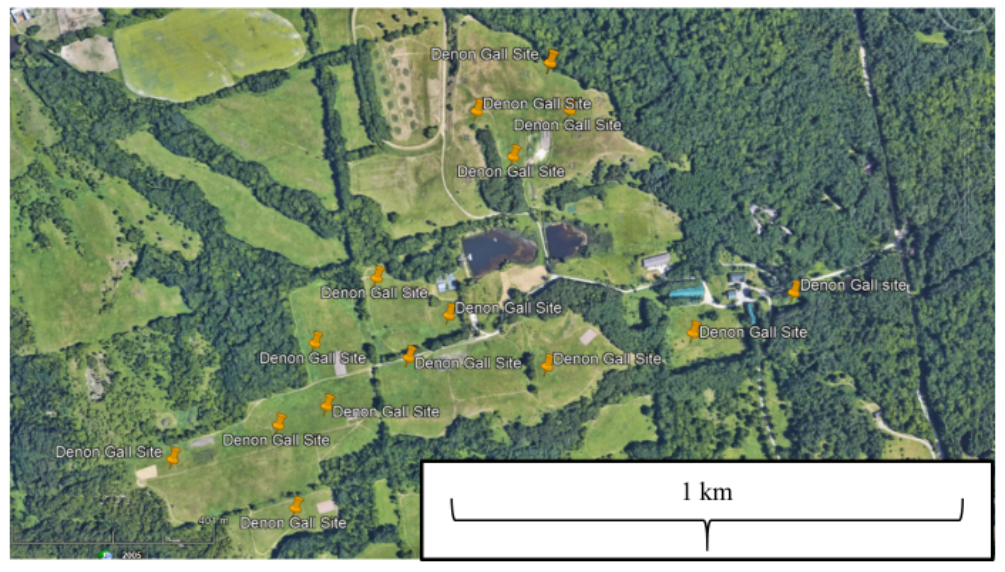

Figure S1: A map showing the location of populations at the Koffler Scientific Reserve. Note that populations are widely dispersed across all field habitat at the field station.

\section{References}

1. Ackerly, D. 2003. Canopy gaps to climate change: extreme events, ecology, and evolution. The New Phytologist 160: 2-4. 
2. Nunez M, Logares R. 2012. Black Swans in ecology and evolution: The importance of improbably but highly influential events. Ideas in Ecology and Evolution 5.

3. Anderson S, Branch TA, Cooper AB, Dulvy NK. 2017. Black-swan events in animal populations. Proc. Natl. Acad. Sci. 114: 3252-3257.

4. Pruitt JN, Little AG, Majumdar SJ, Schoener TW, Fisher DN. 2019. Call-to-action: a global consortium for tropical cyclone ecology. TREE.

5. Fey SB, et al. 2015. Recent shifts in the occurrence, cause, and magnitude of animal mass mortality events. Proc. Natl. Acad. Sci. 112L 1083-1088.

6. Little AG, Fisher DN, Schoener TW, Pruitt JN. 2019. Population differences in aggression are shaped by tropical cyclone-induced selection. Nature ecology \& evolution 3: 1294-1297.

7. Shafi MI, Yarranton GA. 1973. Diversity, floristic richness, and species evenness during a secondary (post-fire) succession. Ecology 54: 897-902.

8. Perry DA, Lotan JE. 1979. A model of fire selection for serotiny in lodgepole pine. Evolution 958-968.

9. Start D, Weis AE, Gilbert B. In Press . Ecological and evolutionary stochasticity shape natural selection. The American Naturalist.

10. Abrahamson WG, Sattler JF, McCrea KD, Weis AE. 1989. Variation in selection pressures on the goldenrod gall fly and the competitive interactions of its natural enemies. Oecologia 79: 15-22.

11. Start D, Gilbert B. 2016. Host-parasitoid evolution in a metacommunity. Proc. R. Soc. B. 283: 20160477.

12. Start D. 2018. Keystone individuals alter ecological and evolutionary consumer-resource dynamics. The American Naturalist 191: 277-286.

13. Start D, Bonner C, Weis AE, Gilbert B. 2018. Consumer-resource interactions along urbanization gradients drive natural selection. Evolution 72: 1863-1873.

14. Uhler LD. 1951. Biology and ecology of the goldenrod gall fly,Eurosta solidaginis. Memories of the Cornell University Agricultural Experiment Station 300: 3-51.

15. Craig TP, Itami JK, Horner JD. 2007. Geographic variation in the evolution and coevolution of a tritrophic interaction. Evolution 61: 1137-1152.

16. Weis AE, Kapelinski A. 1994. A path analysis of the ecological factors behind selection. Evolution 48: 734-746.

17. Start D. 2019. Individual and population differences shape species interactions and natural selection. The American Naturalist 194.

18. Start D. 2019. Indirect interactions shape selection in a multispecies food web. The American Naturalist 193: 321-330.

19. Cronin JT, Hyland K, Abrahamson WG. 2001. The pattern, rate, and range of within-patch movement of a stem-galling fly. Ecological Entomology 26: 16-24.

20. Hess MD, Abrahamson WG, Brown JM. 1996. Intraspecific competition in the goldenrod ballgallmakers: larval mortality, adult fitness, ovipositional and host-plant response. American Midland Naturalist 121-133.

21. Lande R, Arnold SJ. 1983. The measurement of selection on correlated characters. Evolution 37: $1210-1226$.

22. Weis AE, Gorman WL. 1990. Measuring selection on reaction norms: an exploration of the EurostaSolidago system. Evolution 44: 820-831.

23. Bates D, Maechler M, Bolker B, Walker S. 2015. Fitting linear-mixed-effects models using lme4. Journal of Statistical Software 67: 1-48.

24. Fox J, Weisberg S. 2011. An R companion to applied regression, Second Edition. Thousand Oaks, CA: Sage.

25. Ledger ME et al. 2013. Extreme climatic events alter aquatic food webs: a synthesis of evidence from a mesocosm drought experiment. Advances in Ecological Research 48: 343-395.

26. Woodward G et al. 2016. The effects of climatic fluctuations and extreme events on running water ecosystems. Phil. Trans. R. Soc. B 371: 20150274.

27. terHorst $\mathrm{CP}$ et al. 2018. Evolution in a community context: trait responses to multiple species 
interactions. The American Naturalist 191: 369-380.

28. Zarnetske PL, Skelly DK, Urban MC. 2012. Biotic multipliers of climate change. Science 336: 15161518.

29. Benkman CW. 2013. Biotic interaction strength and the intensity of selection. Ecology Letters 16: 1054-1060.

30. Arnold SJ, Wade MJ. 1984. On the measurement of natural and sexual selection: theory. Evolution 38:709-719.

31. Saether BE, Engen S. 2015. The concept of fitness in fluctuation environments. TREE 30: 273-281.

32. Holmgren $\mathrm{M}$ et al. 2006. Extreme climatic events shape arid and semiarid ecosystems. Frontiers in Ecology and the Environment 4: 87-95.

33. Fabina NS, Baskett ML, Gross K. 2015. The differential effects of increasing frequency and the magnitude of extreme events on coral populations. Ecological applications 25: 1534-1545. 